\title{
LOW-ENERGY ANTIKAON-NUCLEON ABSORPTION STUDIES BY AMADEUS*
}

K. Piscicchia ${ }^{a, b}$, M. Cargnelli ${ }^{\mathrm{c}}$, C. Curceanu ${ }^{\mathrm{a}}$, R. Del Grande ${ }^{\mathrm{a}}$

L. Fabbiettid ${ }^{\text {d,e }}$, C. Guaraldo ${ }^{a}$, J. Marton ${ }^{c}$, P. Moskal $^{f}$

A. Scordo ${ }^{\mathrm{a}}$, M. Silarskif ${ }^{\mathrm{f}}$, D. Sirghi ${ }^{\mathrm{a}, \mathrm{g}}, \mathrm{M}$. SkURzOK ${ }^{\mathrm{f}}$

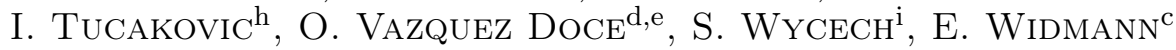

J. ZMESKAL ${ }^{\mathrm{C}}$

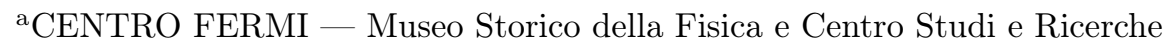
"Enrico Fermi", Roma, Italy

${ }^{b}$ INFN Laboratori Nazionali di Frascati, Frascati (Roma), Italy

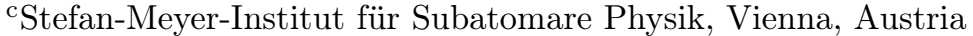

dExcellence Cluster 'Origin and Structure of the Universe', Garching, Germany

ephysik Department E12, Technische Universität München, Garching, Germany

${ }^{\mathrm{f}} \mathrm{M}$. Smoluchowski Institute of Physics, Jagiellonian University, Kraków, Poland

${ }^{g}$ Horia Hulubei National Institute of Physics and Nuclear Engineering

(IFIN-HH), Măgurele, Romania

${ }^{\mathrm{h}}$ Ruđer Bosković Institute, Zagreb, Croatia

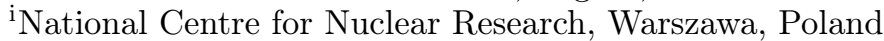

(Received October 2, 2017)

The AMADEUS experiment investigates the low-energy kaon-nuclei hadronic interaction at the DA $\Phi$ NE collider at LNF-INFN. In particular, the study of hyperon resonances formation, following $K^{-}$-induced reactions in light nuclear targets, will be presented. The 0 step of the experiment consisted in the reanalysis of the $2004 / 2005$ KLOE data, exploiting $K^{-}$ absorptions in $\mathrm{H},{ }^{4} \mathrm{He},{ }^{9} \mathrm{Be}$ and ${ }^{12} \mathrm{C}$, leading to the first invariant mass spectroscopic study with very low momentum (about $100 \mathrm{MeV}$ ) in-flight $K^{-}$captures. With AMADEUS step 1, a dedicated pure carbon target was implemented in the central region of the KLOE detector, providing a high statistic sample of pure at-rest $K$ nuclear interaction. The results obtained in the analyses of the hyperon-pion correlated events, searching for the resonant shapes of $\mathrm{Y}^{*}$ states, will be described.

DOI:10.5506/APhysPolBSupp.10.1125

* Presented at "Excited QCD 2017", Sintra, Lisbon, Portugal, May 7-13, 2017. 


\section{Introduction}

The experimental investigation of negative kaon-induced reactions in nuclear matter is a crucial test for the low-energy strangeness QCD. In this sector, the chiral perturbation theory cannot be applied due to the existence of the $\Lambda(1405)$ just few $\mathrm{MeV}$ below the $\bar{K} N$ threshold, which renders this a difficult multi-channel non-perturbative problem. The $\Lambda(1405)$ is a $J^{P}=1 / 2^{-}$ isospin $I=0$ strangeness -1 state, which decays into $(\Sigma \pi)^{0}$ through the strong interaction, whose existence was predicted by Dalitz and Tuan in 1959 [1]; despite the first experimental evidence that came just two years later [2], the nature of the $\Lambda(1405)$ is still controversial. The three-quark picture $(u d s)$ meets some difficulties to explain both the observed $\Lambda(1405)$ mass and the mass splitting with the $\Lambda(1520)$. The low mass of the $\Lambda(1405)$ can be explained in a five-quark picture which, however, predicts more, unobserved, excited baryons. A review of the theoretical and experimental investigation can be found in [3]. The bubble chamber search lead to different positions of the resonance (see, for example, [4-6]) for different charged combinations of the $\Sigma$ and the $\pi$. The isospin interference term is responsible for the different shapes of the $\Sigma^{ \pm} \pi^{\mp}$ invariant mass distributions. The $\Sigma^{0} \pi^{0}$ decay channel, which is free from the $I=1$ contribution and from the isospin interference term, is the cleanest signature of the $\Lambda(1405)$. The experimental $\Sigma^{0} \pi^{0}$ invariant mass shapes, produced in different physical processes [7-9], are also found to strongly differ. This difference is not surprising if seen in the context of the chiral unitary interpretation of the $\Lambda(1405)$ [10-12], which emerges from the interference of two poles, a lower mass pole (about $1390 \mathrm{MeV}$ ) which is mainly coupled to the $\Sigma \pi$ channel and a high mass pole, coupled to the $\bar{K} N$ production channel, located around $1420 \mathrm{MeV}$ (the next to the leading order calculation of the poles masses and widths can be found in [13]). Accordingly, the shape of the $(\Sigma \pi)^{0}$ invariant mass distribution is expected to change depending on the production channel.

The AMADEUS [14] experiment investigates the low-energy $K^{-}$hadronic interaction in light nuclei (e.g. $\mathrm{H},{ }^{4} \mathrm{He},{ }^{9} \mathrm{Be}$ and $\left.{ }^{12} \mathrm{C}\right)$ by exploiting the low momentum (about $127 \mathrm{MeV} / c$ ), almost monochromatic, charged kaons provided by the decay of $\phi$ mesons at-rest at the DA $\Phi$ NE factory [15]. Since the accessible $(\Sigma \pi)^{0}$ invariant mass, in $K^{-} p$ absorption processes, is influenced by the binding energy of the proton in the hosting nucleus, our strategy is to unveil the presence of the high mass pole by exploiting $K^{-}$captures in-flight $[16,17]$. In this case, the kinetic energy of the kaon allows for an investigation of the invariant mass region which is just below the $\bar{K} N$ threshold. The shape of the $(\Sigma \pi)^{0}$ spectra is also distorted by the non-resonant production below threshold. A key issue, which is addressed in the analyses described below, is the investigation of the non-resonant hyperon-pion transition amplitude below threshold. 
The position of the $\Lambda(1405)$ reflects the strength of the $\bar{K} N$ interaction, thus influencing the possible formation of $\bar{K}$ multi-nucleon bound states. For the di-baryonic kaonic bound state $p p K^{-}$, theoretical predictions deliver a wide range of binding energies and widths [18], while the experimental results are contradictory [19-28]. The extraction of $p p K^{-}$signal in $K^{-}$absorption experiments is strongly affected by the yield and the shape of the competing $K^{-}$multi-nucleon absorption processes as clearly evidenced in [29], where the yield of the $K^{-}$double-nucleon absorption, when the produced $\Sigma^{0} p$ pairs are free from final-state interactions [30], was measured for the first time.

\section{Data samples}

The ongoing AMADEUS analyses refer to two data samples. One is represented by the data collected by the KLOE Collaboration [31] during the $2004 / 2005$ data taking, corresponding to $\sim 1.74 \mathrm{fb}^{-1}$. The KLOE detector [32] is used as an active target, the hadronic interaction of negative kaons with the materials of the apparatus being investigated; in particular, $K^{-9} \mathrm{Be}$ absorptions in the DA $\Phi$ NE beryllium thin cylindrical layer and the DA $\Phi \mathrm{NE}$ aluminated beryllium pipe, $K^{-12} \mathrm{C}$ and $K^{-} \mathrm{H}$ absorptions in the KLOE Drift Chamber [33] (DC) inner wall (aluminated carbon fiber), $K^{-4} \mathrm{He}$ in the DC gas. Extremely rich experimental information is contained in this sample, with $K^{-}$hadronic captures both at-rest and in-flight [16].

In order to increase the statistics and as an essential interpretation tool, a high purity carbon target (graphite) was realized in summer 2012 and installed inside the KLOE detector between the beam pipe and the DC inner wall. The geometry of the target was optimized to maximize the kaon stopping power. The total collected integrated luminosity is $\sim 90 \mathrm{pb}^{-1}$. Up to now, we analysed a sample of $37 \mathrm{pb}^{-1}$ reconstructed data.

Details on the events selection and particle identification for the channels under investigation are given in [14].

\section{3. $Y \pi$ resonant and non-resonant production and the shape of the $\Lambda(1405)$}

When extracting the $\Lambda(1405)$ shape from $K^{-}$induced reactions in light nuclear targets (see, for example, [34]) the hyperon-pion spectroscopy is influenced by the energy threshold, imposed by the last nucleon binding energy. The $m_{\Sigma \pi}$ invariant mass threshold is about $1412 \mathrm{MeV}$ and $1416 \mathrm{MeV}$, for $K^{-}$capture at-rest in ${ }^{4} \mathrm{He}$ and ${ }^{12} \mathrm{C}$ respectively, thus the $K^{-}$absorption at-rest is not sensitive to the $\Lambda(1405)$ high mass pole. The $\bar{K} N$ subthreshold region is accessible by exploiting $K^{-} N$ absorptions in-flight. For a mean kaon momentum of $100 \mathrm{MeV} / c$, the $m_{\Sigma \pi}$ threshold is shifted upwards by about $10 \mathrm{MeV}$. Another bias to be considered is represented by 
the non-resonant $K^{-} N \rightarrow Y \pi$ transition. The corresponding $m_{Y \pi}$ invariant masses spectra are narrow (of the order of $10 \mathrm{MeV}$ ) and peaked below the $\bar{K} N$ threshold. The $\Lambda \pi$ and $\Sigma \pi$ non-resonant transition amplitudes, for $K^{-}$capture in light nuclear targets, was never measured. The $\Lambda$ and $\pi^{-}$ kinematic distributions for $K^{-}$captures in ${ }^{4} \mathrm{He}$, both at-rest and in-flight, were calculated in [35]. The momentum probability distribution functions of the emerging hyperon-pion pairs, following $K^{-} n$ absorptions, are expressed in terms of the $K^{-} n$ transition amplitudes: the isospin $I=1 S$-wave nonresonant amplitude $\left(\left|f^{\mathrm{nr}}\right|\right)$ and the resonant $I=1 P$-wave amplitude, dominated by the $\Sigma^{-}(1385)$. Since the resonant amplitude is well-known from direct experiments, the measured total momentum distributions can be used to extract the non-resonant $\left|f^{\mathrm{nr}}\right|$ amplitude module below the $\bar{K} N$ threshold. The goal of the ongoing analyses is to measure the contributions and the shapes of the non-resonant $\Lambda \pi$ and $\Sigma \pi$ productions. The knowledge of the $(\Sigma \pi)^{0}$ isospin $I=0$ non-resonant transition amplitude will allow to disentangle the resonant $\Lambda(1405)$ shape. Preliminary $\Sigma^{+} \pi^{-}$invariant mass spectra, from $K^{-}$captures in the wall of the KLOE DC, not background subtracted nor acceptance corrected, are shown in Fig. 1. The thin black/red curve refers to $K^{-}$absorptions on hydrogen, thin grey/green and thick grey/violet distributions refer to $K^{-}$captures in-flight and at-rest in ${ }^{12} \mathrm{C}$ respectively, the thick black/blue line is the sum of thin grey/green and thick grey/violet distributions. The thin black/red distribution reflects the non-resonant $K^{-} \mathrm{H}$ absorption in-flight, which corresponds to a narrow invariant mass shape peaked below the mass threshold $m_{K}+m_{p}+\left\langle p_{K}^{2}\right\rangle / 2 m_{K}$.

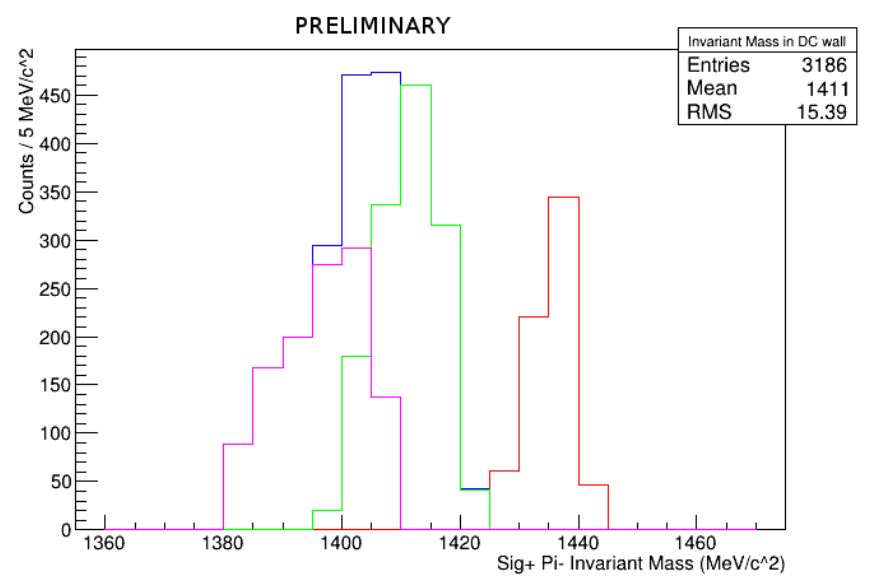

Fig. 1. (Colour on-line) $m_{\Sigma \pi}$ invariant mass distributions in-flight (thin grey/green) and at-rest (thick grey/violet) in ${ }^{12} \mathrm{C}$. Thick black/blue is the sum of green and violet curves. The thin black/red curve refers to $K^{-}$absorptions on hydrogen. 
The measured invariant mass of the $\Sigma^{0} \pi^{0}$ pairs is plotted in Fig. 2 as a function of the $\pi^{0}$ momentum (see Ref. [36]). Figure 2, left refers to $K^{-}$ absorptions in the inner wall of the KLOE DC, this sample then contains both $K^{-}$captures at-rest and in-flight. In Fig. 2, right, the same $m_{\Sigma^{0} \pi^{0}}$ versus $p_{\pi^{0}}$ scatterplot is shown for $K^{-}$absorptions at-rest in the carbon target. The $\pi^{0}$ momentum is a useful variable to distinguish among the two capture processes, as the pion gains great part of the kinetic energy when the $K^{-}$interacts in-flight. An enhancement is evident in Fig. 2, left corresponding to the in-flight events, which is correlated with invariant masses $m_{\Sigma^{0} \pi^{0}}$ centred at about $1425 \mathrm{MeV}$, where the high mass pole of the $\Lambda(1405)$ is expected. A spectroscopic study of the kinematic region ranging between the at-rest energy threshold, and the $\bar{K} N$ threshold, opened by the low momentum in-flight capture process, will allow to clarify the nature of the high mass $\Lambda(1405)$ pole.
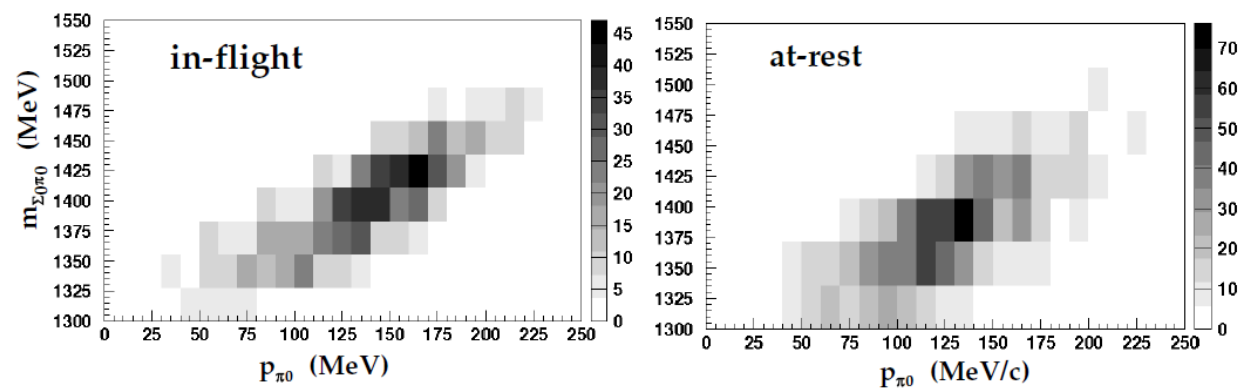

Fig. 2. $m_{\Sigma^{0} \pi^{0}}$ versus $p_{\pi}^{0}$ for $K^{-}$captures in the KLOE DC wall (left) and pure carbon graphite target (right).

\section{Conclusions and perspectives}

We presented in this work a preliminary investigation of the resonant versus non-resonant hyperon-pion production, following $K^{-}$absorptions in ${ }^{4} \mathrm{He}$ and ${ }^{12} \mathrm{C}$. It is shown that the process of low momentum $K^{-}$capture in-flight, opening the $m_{\Sigma \pi}$ window which ranges among the kinematic threshold atrest and the $\bar{K} N$ threshold, is suitable for the search and the chracterization of the high mass pole of the $\Lambda(1405)$.

A feasibility study for the realization of a dedicated AMADEUS experimental setup $[37,38]$ is presently ongoing in order to deepen and extend the low-energy anti-kaon nuclei interaction studies and obtain fundamental input for the investigation of QCD with strangeness. 
We acknowledge the KLOE Collaboration for their support and for having provided us the data and the tools to perform the analysis presented in this paper. We thank as well the DA $\Phi$ NE staff for the excellent working conditions and permanent support. We acknowledge the CENTRO FERMI - Museo Storico della Fisica e Centro Studi e Ricerche "Enrico Fermi", for the project PAMQ. Part of this work was supported by the Austrian Science Fund (FWF): [P24756-N20]; Austrian Federal Ministry of Science and Research BMBWK 650962/0001 VI/2/2009; the Grants-in-Aid for Specially Promoted Research (20002003), MEXT, Japan; Minstero degli Affari Esteri e della Cooperazione Internazionale, Direzione Generale per la Promozione del Sistema Paese (MAECI), Strange Matter project; National Science Centre, Poland (NCN) through grant No. UMO-2016/21/D/ST2/01155.

\section{REFERENCES}

[1] R.H. Dalitz, S.F. Tuan, Phys. Rev. Lett. 2, 425 (1959).

[2] M.H. Alston et al., Phys. Rev. Lett. 6, 698 (1961).

[3] T. Hyodo, D. Jido, Prog. Part. Nucl. Phys. 67, 55 (2012).

[4] O. Braun et al., Nucl. Phys. B 129, 1 (1977).

[5] D.W. Thomas et al., Nucl. Phys. B 56, 15 (1973).

[6] R.J. Hemingway, Nucl. Phys. B 253, 742 (1985).

[7] S. Prakhov et al., Phys Rev. C 70, 03465 (2004).

[8] K. Moriya, R. Schumacher [CLAS Collaboration], AIP Conf. Proc. 1441, 296 (2012).

[9] I. Zychor et al., Phys. Lett. B 660, 167 (2008).

[10] J.A. Oller, U.-G. Meißner, Phys. Lett. B 500, 263 (2001).

[11] T. Hyodo et al., Phys Rev. C 68, 018201 (2003).

[12] D. Jido et al., Nucl. Phys. A 725, 181 (2003).

[13] Y. Ikeda, T. Hyodo, W. Weise, Nucl. Phys. A 881, 98 (2012).

[14] C. Curceanu et al., Acta Phys. Pol. B 46, 203 (2015).

[15] G.V. Vignola et al., Conf. Proc. C 1993, 930517 (1993).

[16] K. Piscicchia et al., PoS Bormio2013, 034 (2013).

[17] A. Scordo et al., PoS Bormio2014, 039 (2014).

[18] T. Yamazaki et al., Phys Rev. C 76, 045201 (2007); N.V. Shevchenko et al., Phys. Rev. Lett. 98, 082301 (2007); P. Bicudo, Phys. Rev. D 76, 031502 (2007); A. Doté et al., Phys Rev. C 79, 014003 (2009); S. Wycech et al., Phys Rev. C 79, 014001 (2009); Y. Ikeda et al., Phys Rev. C 79, 035201 (2009); N. Barnea et al., Phys. Lett. B 712, 132 (2012); E. Oset et al., Nucl. Phys. A 881, 127 (2012); M. Bayar, E. Oset, Nucl. Phys. A 914, 349 (2013).

[19] G. Agakishiev et al. [HADES Collaboration], Phys. Lett. B 742, 242 (2015). 
[20] M. Agnello et al. [FINUDA Collaboration], Phys. Rev. Lett. 94, 212303 (2005).

[21] T. Yamazaki et al., Phys. Rev. Lett. 104, 132502 (2010).

[22] Y. Ichikawa et al., Prog. Theor. Exp. Phys. 2015, 021001 (2015).

[23] M. Silarski et al., Phys Rev. C 88, 025205 (2013).

[24] A.O. Tokiyasu et al., Phys. Lett. B 728, 616 (2014).

[25] L. Fabbietti et al., Nucl. Phys. A 914, 60 (2013).

[26] T. Hashimoto et al., Prog. Theor. Exp. Phys. 2015, 061001 (2015).

[27] M. Iwasaki, EPJ Web Conf. 130, 01023 (2016).

[28] Y. Sada et al., Prog. Theor. Exp. Phys. 2016, $051 D 01$ (2016).

[29] O. Vazquez Doce et al., Phys. Lett. B 758, 134 (2016).

[30] S. Sewerin et al., Phys. Rev. Lett. 83, 682 (1999).

[31] F. Bossi et al. [KLOE Collaboration], Riv. Nuovo Cim. 31, 531 (2008).

[32] F. Ambrosino et al., Nucl. Instrum. Methods Phys. Res. A 534, 403 (2004).

[33] M. Adinolfi et al. [KLOE Collaboration], Nucl. Instrum. Methods Phys. Res. A 488, 51 (2002).

[34] J. Esmaili et al., Phys. Lett. B 686, 23 (2010).

[35] K. Piscicchia, S. Wycech, C. Curceanu, Nucl. Phys. A 954, 75 (2016).

[36] K. Piscicchia, Ph.D. Thesis, 2013, Lambda(1405) Measurement Through the Decay to $\Sigma^{0} \pi^{0}$, Resulting from $K^{-}$Meson Absorption on ${ }^{4} \mathrm{He}$ and ${ }^{12} \mathrm{C}$, with the KLOE Detector, http://www.infn.it/thesis/thesis_dettaglio.php?tid=7097

[37] M. Bazzi et al., Nucl. Instrum. Methods Phys. Res. A 671, 125 (2012).

[38] M. Bazzi et al., JINST 8, T11003 (2013). 